\title{
Measurements of the branching fractions $\mathcal{B}\left(B^{-} \rightarrow \bar{\Lambda}_{c}^{-} \Xi_{c}^{\prime \mathbf{0}}\right)$, $\mathcal{B}\left(B^{-} \rightarrow \bar{\Lambda}_{c}^{-} \Xi_{c}(2645)^{0}\right)$ and $\mathcal{B}\left(B^{-} \rightarrow \bar{\Lambda}_{c}^{-} \Xi_{c}(2790)^{0}\right)$
}

Y. Li, ${ }^{2}$ Y. B. Li, ${ }^{70}$ C. P. Shen $\odot,{ }^{10}$ I. Adachi, ${ }^{17,13}$ H. Aihara, ${ }^{84}$ D. M. Asner, ${ }^{3}$ H. Atmacan, ${ }^{76}$ V. Aulchenko,,${ }^{4,66}$ R. Ayad ${ }^{79}$ V. Babu, ${ }^{8}$ I. Badhrees, ${ }^{79,35}$ A. M. Bakich, ${ }^{78}$ P. Behera ${ }^{24}$ T. Bilka, ${ }^{5}$ J. Biswal, ${ }^{32}$ A. Bobrov, ${ }^{4,66}$ G. Bonvicini, ${ }^{88}$ A. Bozek, ${ }^{62}$ M. Bračko, ${ }^{48,32}$ T. E. Browder, ${ }^{16}$ M. Campajola ${ }^{29,57}$ L. Cao, ${ }^{33}$ D. Červenkov, ${ }^{5}$ P. Chang, ${ }^{61}$ A. Chen, ${ }^{59}$ B. G. Cheon, ${ }^{15}$ K. Chilikin, ${ }^{42}$ H. E. Cho, ${ }^{15}$ K. Cho, ${ }^{36}$ S.-K. Choi,${ }^{14}$ Y. Choi ${ }^{77}$ D. Cinabro ${ }^{88}$ S. Cunliffe, ${ }^{8}$ G. De Nardo, ${ }^{29,57}$ F. Di Capua, ${ }^{29,57}$ S. Di Carlo, ${ }^{40}$ Z. Doležal, ${ }^{5}$ T. V. Dong, ${ }^{10}$ S. Eidelman, ${ }^{4,66,42}$ J. E. Fast, ${ }^{68}$ T. Ferber, ${ }^{8}$ D. Ferlewicz, ${ }^{50}$ B. G. Fulsom, ${ }^{68}$ R. Garg, ${ }^{69}$ V. Gaur, ${ }^{87}$ N. Gabyshev, ${ }^{4,66}$ A. Garmash, ${ }^{4,66}$ A. Giri, ${ }^{23}$ P. Goldenzweig, ${ }^{33}$ B. Golob, ${ }^{44,32}$ B. Grube, ${ }^{81}$ T. Hara, ${ }^{17,13}$ K. Hayasaka, ${ }^{64}$ H. Hayashii ${ }^{58}$ W.-S. Hou, ${ }^{61}$ C.-L. Hsu, ${ }^{78}$ T. Iijima, ${ }^{56,55}$ K. Inami, ${ }^{55}$ G. Inguglia, ${ }^{27}$ A. Ishikawa, ${ }^{17,13}$ R. Itoh ${ }^{17,13}$ M. Iwasaki ${ }^{67}$ Y. Iwasaki, ${ }^{17}$ W. W. Jacobs ${ }^{25}$ S. Jia, ${ }^{2}$ Y. Jin,${ }^{84}$ K. K. Joo, ${ }^{6}$ K. H. Kang, ${ }^{39}$ G. Karyan, ${ }^{8}$ H. Kichimi ${ }^{17}$ C. H. Kim, ${ }^{15}$ D. Y. Kim, ${ }^{75}$ S. H. Kim,${ }^{15}$ K. Kinoshita, ${ }^{7}$ P. Kodyš, ${ }^{5}$ S. Korpar, ${ }^{48,32}$ R. Kroeger, ${ }^{51}$ P. Krokovny, ${ }^{4,66}$ T. Kuhr, ${ }^{45}$ R. Kulasiri, ${ }^{34}$ A. Kuzmin, ${ }^{4,66}$ Y.-J. Kwon, ${ }^{90}$ K. Lalwani, ${ }^{47}$ J. S. Lange, ${ }^{11}$ I. S. Lee ${ }^{15}$ S. C. Lee, ${ }^{39}$ P. Lewis, ${ }^{16}$ C. H. Li ${ }^{43}$ L. K. Li ${ }^{26}$ L. Li Gioi,${ }^{49}$ J. Libby, ${ }^{24}$ K. Lieret, ${ }^{45}$ D. Liventsev ${ }^{87,17}$ C. MacQueen ${ }^{50}$ M. Masuda, ${ }^{83}$ T. Matsuda, ${ }^{52}$ D. Matvienko, ${ }^{4,66,42}$ M. Merola, ${ }^{29,57}$ H. Miyata ${ }^{64}$ R. Mizuk, ${ }^{42,54}$ R. Mussa, ${ }^{30}$ M. Nakao ${ }^{17,13}$ K. J. Nath ${ }^{22}$ M. Nayak, ${ }^{88,17}$ M. Niiyama, ${ }^{38}$ N. K. Nisar, ${ }^{71}$ S. Nishida, ${ }^{17,13}$ K. Nishimura, ${ }^{16}$ S. Ogawa, ${ }^{82}$ H. Ono, ${ }^{63,64}$ Y. Onuki ${ }^{84}$ P. Oskin, ${ }^{42}$ P. Pakhlov, ${ }^{42,53}$ G. Pakhlova, ${ }^{42,54}$ T. Pang, ${ }^{71}$ S. Pardi, ${ }^{29}$ H. Park, ${ }^{39}$ S.-H. Park, ${ }^{90}$ S. Patra, ${ }_{33}^{21}$ S. Paul, ${ }^{81}$ T. K. Pedlar, ${ }^{46}$ R. Pestotnik, ${ }^{32}$ L. E. Piilonen, ${ }^{87}$ T. Podobnik, ${ }^{44,32}$ V. Popov, ${ }^{42,54}$ E. Prencipe ${ }^{19}$ M. T. Prim ${ }^{33}$ M. Röhrken, ${ }^{8}$ A. Rostomyan, ${ }^{8}$ N. Rout, ${ }^{24}$ G. Russo, ${ }^{57}$ D. Sahoo, ${ }^{80}$ Y. Sakai, ${ }^{17,13}$ S. Sandilya, ${ }^{7}$ L. Santelj, ${ }^{17}$ V. Savinov, ${ }^{71}$ O. Schneider ${ }^{41}$ G. Schnell, ${ }^{1,20}$ C. Schwanda, ${ }^{27}$ Y. Seino, ${ }^{64}$ K. Senyo, ${ }^{89}$ M. E. Sevior,${ }^{50}$ J.-G. Shiu, ${ }^{61}$ B. Shwartz, ${ }^{4,66}$ A. Sokolov ${ }^{28}{ }^{2}$ E. Solovieva, ${ }^{42}$ S. Stanič ${ }^{65}$ M. Starič ${ }^{32}$ Z. S. Stottler, ${ }^{87}$ M. Sumihama, ${ }^{12}$ T. Sumiyoshi, ${ }^{86}$ W. Sutcliffe, ${ }^{33}$ M. Takizawa, ${ }^{74,18,72}$ K. Tanida, ${ }^{31}$ F. Tenchini, ${ }^{8}$ K. Trabelsi, ${ }^{40}$ M. Uchida,${ }^{85}$ T. Uglov,${ }^{42,54}$ Y. Unno, ${ }^{15}$ S. Uno, ${ }^{17,13}$ R. Van Tonder, ${ }^{33}$ G. Varner, ${ }^{16}$ A. Vinokurova, ${ }^{4,66}$ C. H. Wang, ${ }^{60}$ M.-Z. Wang, ${ }^{61}$ X. L. Wang, ${ }^{10}$ M. Watanabe,${ }^{64}$ E. Won, ${ }^{37}$ S. B. Yang, ${ }^{37}$ H. Ye, ${ }^{8}$ J. Yelton, ${ }^{9}$ J. H. Yin, ${ }^{26}$ C. Z. Yuan, ${ }^{26}$ Z. P. Zhang, ${ }^{73}$ V. Zhilich, ${ }^{4,66}$ V. Zhukova, ${ }^{42}$ and V. Zhulanov ${ }^{4,66}$

(Belle Collaboration)

\author{
${ }^{1}$ University of the Basque Country UPV/EHU, 48080 Bilbao \\ ${ }^{2}$ Beihang University, Beijing 100191 \\ ${ }^{3}$ Brookhaven National Laboratory, Upton, New York 11973 \\ ${ }^{4}$ Budker Institute of Nuclear Physics SB RAS, Novosibirsk 630090 \\ ${ }^{5}$ Faculty of Mathematics and Physics, Charles University, 12116 Prague \\ ${ }^{6}$ Chonnam National University, Gwangju 61186 \\ ${ }^{7}$ University of Cincinnati, Cincinnati, Ohio 45221 \\ ${ }^{8}$ Deutsches Elektronen-Synchrotron, 22607 Hamburg \\ ${ }^{9}$ University of Florida, Gainesville, Florida 32611 \\ ${ }^{10}$ Key Laboratory of Nuclear Physics and Ion-beam Application (MOE) and Institute of Modern Physics, \\ Fudan University, Shanghai 200443 \\ ${ }^{11}$ Justus-Liebig-Universität Gießen, 35392 Gießen \\ ${ }^{12}$ Gifu University, Gifu 501-1193 \\ ${ }^{13}$ SOKENDAI (The Graduate University for Advanced Studies), Hayama 240-0193 \\ ${ }^{14}$ Gyeongsang National University, Jinju 52828 \\ ${ }^{15}$ Department of Physics and Institute of Natural Sciences, Hanyang University, Seoul 04763 \\ ${ }^{16}$ University of Hawaii, Honolulu, Hawaii 96822 \\ ${ }^{17}$ High Energy Accelerator Research Organization (KEK), Tsukuba 305-0801 \\ ${ }^{18}$ J-PARC Branch, KEK Theory Center, High Energy Accelerator Research Organization (KEK), \\ Tsukuba 305-0801 \\ ${ }^{19}$ Forschungszentrum Jülich, 52425 Jülich \\ ${ }^{20}$ IKERBASQUE, Basque Foundation for Science, 48013 Bilbao \\ ${ }^{21}$ Indian Institute of Science Education and Research Mohali, SAS Nagar 140306 \\ ${ }^{22}$ Indian Institute of Technology Guwahati, Assam 781039 \\ ${ }^{23}$ Indian Institute of Technology Hyderabad, Telangana 502285 \\ ${ }^{24}$ Indian Institute of Technology Madras, Chennai 600036 \\ ${ }^{25}$ Indiana University, Bloomington, Indiana 47408 \\ ${ }^{26}$ Institute of High Energy Physics, Chinese Academy of Sciences, Beijing 100049
}


${ }^{27}$ Institute of High Energy Physics, Vienna 1050

${ }^{28}$ Institute for High Energy Physics, Protvino 142281

${ }^{29}$ INFN-Sezione di Napoli, 80126 Napoli

${ }^{30}$ INFN-Sezione di Torino, 10125 Torino

${ }^{31}$ Advanced Science Research Center, Japan Atomic Energy Agency, Naka 319-1195

${ }^{32}$ J. Stefan Institute, 1000 Ljubljana

${ }^{33}$ Institut für Experimentelle Teilchenphysik, Karlsruher Institut für Technologie, 76131 Karlsruhe

${ }^{34}$ Kennesaw State University, Kennesaw, Georgia 30144

${ }^{35}$ King Abdulaziz, City for Science and Technology, Riyadh 11442

${ }^{36}$ Korea Institute of Science and Technology Information, Daejeon 34141

${ }^{37}$ Korea University, Seoul 02841

${ }^{38}$ Kyoto University, Kyoto 606-8502

${ }^{39}$ Kyungpook National University, Daegu 41566

${ }^{40}$ LAL, Univ. Paris-Sud, CNRS/IN2P3, Université Paris-Saclay, Orsay 91898

${ }^{41}$ École Polytechnique Fédérale de Lausanne (EPFL), Lausanne 1015

${ }^{42}$ P.N. Lebedev Physical Institute of the Russian Academy of Sciences, Moscow 119991

${ }^{43}$ Liaoning Normal University, Dalian 116029

${ }^{44}$ Faculty of Mathematics and Physics, University of Ljubljana, 1000 Ljubljana

${ }^{45}$ Ludwig Maximilians University, 80539 Munich

${ }^{46}$ Luther College, Decorah, Iowa 52101

${ }^{47}$ Malaviya National Institute of Technology Jaipur, Jaipur 302017

${ }^{48}$ University of Maribor, 2000 Maribor

${ }^{49}$ Max-Planck-Institut für Physik, 80805 München

${ }^{50}$ School of Physics, University of Melbourne, Victoria 3010

${ }^{51}$ University of Mississippi, University, Mississippi 38677

${ }^{52}$ University of Miyazaki, Miyazaki 889-2192

${ }^{53}$ Moscow Physical Engineering Institute, Moscow 115409

${ }^{54}$ Moscow Institute of Physics and Technology, Moscow Region 141700

${ }^{55}$ Graduate School of Science, Nagoya University, Nagoya 464-8602

${ }^{56}$ Kobayashi-Maskawa Institute, Nagoya University, Nagoya 464-8602

${ }^{57}$ Università di Napoli Federico II, 80055 Napoli

${ }^{58}$ Nara Women's University, Nara 630-8506

${ }^{59}$ National Central University, Chung-li 32054

${ }^{60}$ National United University, Miao Li 36003

${ }^{61}$ Department of Physics, National Taiwan University, Taipei 10617

${ }^{62}$ H. Niewodniczanski Institute of Nuclear Physics, Krakow 31-342

${ }^{63}$ Nippon Dental University, Niigata 951-8580

${ }^{64}$ Niigata University, Niigata 950-2181

${ }^{65}$ University of Nova Gorica, 5000 Nova Gorica

${ }^{66}$ Novosibirsk State University, Novosibirsk 630090

${ }^{67}$ Osaka City University, Osaka 558-8585

${ }^{68}$ Pacific Northwest National Laboratory, Richland, Washington 99352

${ }^{69}$ Panjab University, Chandigarh 160014

${ }^{70}$ Peking University, Beijing 100871

${ }^{71}$ University of Pittsburgh, Pittsburgh, Pennsylvania 15260

${ }^{72}$ Theoretical Research Division, Nishina Center, RIKEN, Saitama 351-0198

${ }^{73}$ University of Science and Technology of China, Hefei 230026

${ }^{74}$ Showa Pharmaceutical University, Tokyo 194-8543

${ }^{75}$ Soongsil University, Seoul 06978

${ }^{76}$ University of South Carolina, Columbia, South Carolina 29208

${ }^{77}$ Sungkyunkwan University, Suwon 16419

${ }^{78}$ School of Physics, University of Sydney, New South Wales 2006

${ }^{79}$ Department of Physics, Faculty of Science, University of Tabuk, Tabuk 71451

${ }^{80}$ Tata Institute of Fundamental Research, Mumbai 400005

${ }^{81}$ Department of Physics, Technische Universität München, 85748 Garching

${ }^{82}$ Toho University, Funabashi 274-8510

${ }^{83}$ Earthquake Research Institute, University of Tokyo, Tokyo 113-0032

${ }^{84}$ Department of Physics, University of Tokyo, Tokyo 113-0033

${ }^{85}$ Tokyo Institute of Technology, Tokyo 152-8550

${ }^{86}$ Tokyo Metropolitan University, Tokyo 192-0397 


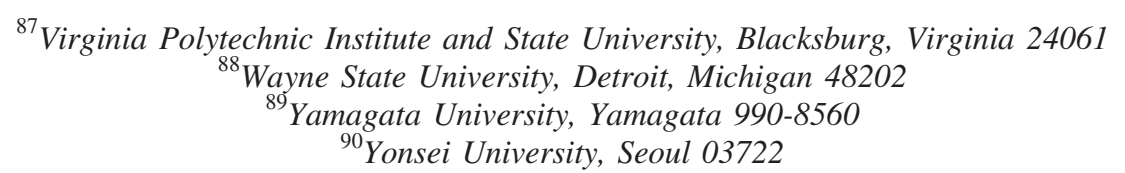

(Received 28 November 2019; published 30 December 2019)

\begin{abstract}
Using the data sample of $711 \mathrm{fb}^{-1}$ of $\Upsilon(4 S)$ on-resonance data taken by the Belle detector at the KEKB asymmetric-energy electron-positron collider, we present the first measurements of branching fractions of the decays $B^{-} \rightarrow \bar{\Lambda}_{c}^{-} \Xi_{c}^{\prime 0}, B^{-} \rightarrow \bar{\Lambda}_{c}^{-} \Xi_{c}(2645)^{0}$, and $B^{-} \rightarrow \bar{\Lambda}_{c}^{-} \Xi_{c}(2790)^{0}$. The signal yields for these decays are extracted from the recoil mass spectrum of the system recoiling against $\bar{\Lambda}_{c}^{-}$baryons in selected $B^{-}$ candidates. The branching fraction of $B^{-} \rightarrow \bar{\Lambda}_{c}^{-} \Xi_{c}(2790)^{0}$ is measured to be $(1.1 \pm 0.4 \pm 0.2) \times 10^{-3}$, where the first uncertainty is statistical and the second is systematic. The $90 \%$ credibility level upper limits on $\mathcal{B}\left(B^{-} \rightarrow \bar{\Lambda}_{c}^{-} \Xi_{c}^{\prime 0}\right)$ and $\mathcal{B}\left(B^{-} \rightarrow \bar{\Lambda}_{c}^{-} \Xi_{c}(2645)^{0}\right)$ are determined to be $6.5 \times 10^{-4}$ and $7.9 \times 10^{-4}$, respectively.
\end{abstract}

DOI: $10.1103 /$ PhysRevD.100.112010

\section{INTRODUCTION}

Charm physics is of high interest mainly due to the fact that the charm system provides a unique laboratory to study the subtle interplay of strong and weak interactions. Baryons with one charm quark and two light quarks are called charmed baryons. In the heavy quark symmetry (HQS) approach [1], the two light quarks are regarded as a light diquark. As chiral symmetry and HQS can provide some qualitative insights into their dynamics, the study of charmed baryons plays an important role in improving our understanding of the quark confinement mechanism. The $\Xi_{c}$ charmed baryon states contain one charm quark, one strange quark, and one up or down quark. The ground state $\Xi_{c}^{0}$ and $\Xi_{c}^{+}$baryons, which have spin-parity quantum numbers $J^{P}=\frac{1}{2}^{+}$and no internal orbital angular momentum, are the only members of the group that decay weakly. A growing number of excited $\Xi_{c}$ states have been observed in different experiments [2]. However, much is still unknown about them. Many theoretical approaches have been used to study the excitation spectrum of $\Xi_{c}$ baryons and their decays. These models include quark models, heavy quark $1 / m_{Q}$ and $1 / N_{c}$ expansions, the coupled channel model, and QCD sum rules [3-7]. Through these QCD-inspired relativistic theories, the mass spectrum of excited $\Xi_{c}$ can be predicted. Recently, the masses and intrinsic widths of isodoublets of the excited $\Xi_{c}$ states $\Xi_{c}^{\prime}$, $\Xi_{c}(2645), \Xi_{c}(2790), \Xi_{c}(2815)$, and $\Xi_{c}(2980)$ were measured more precisely by Belle by analyzing their exclusive decays [8].

Published by the American Physical Society under the terms of the Creative Commons Attribution 4.0 International license. Further distribution of this work must maintain attribution to the author(s) and the published article's title, journal citation, and DOI. Funded by SCOAP.
The decay $B^{-} \rightarrow \bar{\Lambda}_{c}^{-} \Xi_{c}^{0}$ proceeds via $b \rightarrow c \bar{c} s$ transition and has a relatively large branching fraction of the order of $10^{-3}[2,9]$. Therefore, a $B$-meson factory provides an experimental research platform to investigate the $\Xi_{c}^{0}$ excitation spectrum exclusively through $B^{-} \rightarrow \bar{\Lambda}_{c}^{-}+$anything decays. This makes it possible to search for missing excited $\Xi_{c}^{0}$ states. In addition, the measurement of their production rates is a good test for the theoretical calculation of $b \rightarrow$ $c \bar{c} s$ transition processes.

In this paper, we measure the branching fractions of $B^{-} \rightarrow \bar{\Lambda}_{c}^{-} \Xi_{c}^{* 0}$ decays based on data collected by the Belle detector at the KEKB asymmetric-energy electron-positron collider. Here and throughout this paper, $\Xi_{c}^{* 0}$ represents $\Xi_{c}^{\prime 0}$, $\Xi_{c}(2645)^{0}$, and $\Xi_{c}(2790)^{0}$ unless otherwise stated. We use a full hadron-reconstruction algorithm [10] to tag a $B^{+}$ signal, denoted $B_{\text {tag }}^{+}$, and then reconstruct a $\bar{\Lambda}_{c}^{-}$using its $\bar{p} K^{+} \pi^{-}$and $\bar{p} K_{S}^{0}\left(K_{S}^{0} \rightarrow \pi^{+} \pi^{-}\right)$decay modes [11] from the remaining tracks. We search for peaks in the invariant mass spectrum of the system recoiling against the $\bar{\Lambda}_{c}^{-}$baryons in the selected $B^{-} \rightarrow \bar{\Lambda}_{c}^{-} \Xi_{c}^{* 0}$ candidates, to extract $\Xi_{c}^{* 0}$ signal yields, from which we calculate the branching fractions of $B^{-} \rightarrow \bar{\Lambda}_{c}^{-} \Xi_{c}^{* 0}$.

\section{DATA SAMPLE AND THE BELLE DETECTOR}

This analysis utilizes a data sample of $711 \mathrm{fb}^{-1}$ collected at the $\Upsilon(4 S)$ on-resonance corresponding to $(772 \pm 11) \times$ $10^{6} B \bar{B}$ pairs. All the data were collected with the Belle detector [12] operating at the KEKB asymmetric-energy $e^{+} e^{-}$collider [13]. The Belle detector is described in detail in Ref. [12]. It is a large solid-angle magnetic spectrometer consisting of a silicon vertex detector, a 50-layer central drift chamber (CDC), an array of aerogel threshold Cherenkov counters (ACC), a barrel-like arrangement of time-of-flight scintillation counters (TOF), and an electromagnetic calorimeter comprised of CsI(TI) crystals located 
inside a superconducting solenoid coil that provides a $1.5 T$ magnetic field. An iron flux return placed outside the coil is instrumented to detect $K_{L}^{0}$ mesons and to identify muons.

To optimize the signal selection criteria and to determine the signal reconstruction efficiency, Monte Carlo (MC) signal events are generated using EvtGen [14], while $\Xi_{c}^{* 0}$ inclusive decays are simulated using PYTHIA [15]. These events are processed by a detector simulation based on GEANT3 [16]. Inclusive MC samples of $\Upsilon(4 S) \rightarrow B \bar{B}$ $\left(B=B^{+}\right.$or $\left.B^{0}\right)$ and $e^{+} e^{-} \rightarrow q \bar{q}(q=u, d, s, c)$ events at $\sqrt{s}=10.58 \mathrm{GeV}$ corresponding to more than three times the integrated luminosity of the data are used to check the backgrounds.

\section{COMMON EVENT SELECTION CRITERIA}

To select the signal candidates, the following event selection criteria are applied. For well-reconstructed charged tracks, except those from $K_{S}^{0} \rightarrow \pi^{+} \pi^{-}$decays, the impact parameters perpendicular to and along the beam direction with respect to the nominal interaction point (IP) are required to be less than 1 and $4 \mathrm{~cm}$, respectively, and the transverse momentum in the laboratory frame is required to be larger than $0.1 \mathrm{GeV} / c$. For the particle identification of a well-reconstructed charged track, information from different detector subsystems, including specific ionization in the $\mathrm{CDC}$, time measurement in the TOF, and the response of the ACC, is combined to form a likelihood $\mathcal{L}_{i}$ [17] for particle species $i$, where $i=\pi, K$, or $p$. Tracks with $R_{K}=$ $\mathcal{L}_{K} /\left(\mathcal{L}_{K}+\mathcal{L}_{\pi}\right)<0.4$ are identified as pions with an efficiency of $97 \%$, while $5 \%$ of kaons are misidentified as pions; tracks with $R_{K}>0.6$ are identified as kaons with an efficiency of $95 \%$, while $4 \%$ of pions are misidentified as kaons. A track with $\mathcal{R}_{p}^{\pi}=\mathcal{L}_{p} /\left(\mathcal{L}_{p}+\mathcal{L}_{\pi}\right)>0.6$ and $\mathcal{R}_{p}^{K}=\mathcal{L}_{p} /\left(\mathcal{L}_{p}+\mathcal{L}_{K}\right)>0.6$ is identified as an (anti)proton with an efficiency of about $97 \%$; fewer than $1 \%$ of the pions and kaons are misidentified as (anti)protons. With the exception of those from $K_{S}^{0}$ decays, all charged tracks are required to be positively identified by the above procedure.

The $K_{S}^{0}$ candidates are first reconstructed from pairs of oppositely charged tracks, which are treated as pions, with a production vertex significantly separated from the average IP, then selected using a multivariate analysis using an artificial neural network [18] based on two sets of input variables [19].

Applying a full reconstruction algorithm of hadronic $B$ meson decays [10] which uses a multivariate analysis based on the NeuroBayes package, we reconstruct $B_{\text {tag }}^{+}$candidates. Each $B_{\text {tag }}^{+}$candidate has an output value $O_{\mathrm{NN}}$ from the multivariate analysis ranging from 0 to 1 . A candidate with larger $O_{\mathrm{NN}}$ is more likely to be a true $B$ meson. If multiple $B_{\text {tag }}^{+}$candidates are found in an event, only the candidate with largest $O_{\mathrm{NN}}$ is selected. To improve the purity of the tagged side, we take $O_{\mathrm{NN}}>0.001, M_{\mathrm{bc}}^{\mathrm{tag}}>5.27 \mathrm{GeV} / c^{2}$, and $\left|\Delta E^{\mathrm{tag}}\right|<0.04 \mathrm{GeV}$ as the signal region. Here, $M_{\mathrm{bc}}^{\mathrm{tag}} \equiv$ $\sqrt{E_{\text {beam }}^{2}-\left(\sum_{i} \vec{p}_{i}^{\mathrm{tag}}\right)^{2}}$ and $\Delta E^{\mathrm{tag}} \equiv \sum_{i} E_{i}^{\mathrm{tag}}-E_{\text {beam }}$, where $E_{\text {beam }} \equiv \sqrt{s} / 2$ is the beam energy and $\left(E_{i}^{\mathrm{tag}}, \vec{p}_{i}^{\mathrm{tag}}\right)$ is the 4momentum of the $B_{\text {tag }}^{+}$daughter $i$ in the $e^{+} e^{-}$center-ofmass system. After reconstructing the $B_{\text {tag }}^{+}$candidate, the $\bar{\Lambda}_{c}^{-} \rightarrow \bar{p} K^{+} \pi^{-}$and $\bar{\Lambda}_{c}^{-} \rightarrow \bar{p} K_{S}^{0}$ decays are reconstructed from the remaining tracks. We perform a fit for the $\bar{\Lambda}_{c}^{-}$ decay vertex and require that $\chi_{\text {vertex }}^{2} /$ n.d.f. $<15$, where n.d.f. is the number of degrees of freedom. The multicombination rate of $\bar{\Lambda}_{c}^{-}$candidates is $21 \%$. If there is more than one $\bar{\Lambda}_{c}^{-}$candidate in an event, the candidate with the smallest $\chi_{\text {vertex }}^{2} /$ n.d.f. is selected. The $\bar{\Lambda}_{c}^{-}$signal region is defined to be $\left|M_{\bar{\Lambda}_{c}^{-}}-m_{\bar{\Lambda}_{c}^{-}}\right|<10 \mathrm{MeV} / c^{2}$ corresponding to about $3 \sigma$, where $\sigma$ denotes the standard deviation. Here, $M_{\bar{\Lambda}_{c}^{-}}$is the reconstructed and $m_{\bar{\Lambda}_{c}^{-}}$is the nominal mass of the $\bar{\Lambda}_{c}^{-}[2]$.

\section{IV. $\Xi_{c}^{\prime 0}, \Xi_{c}(2645)^{0}$, AND $\Xi_{c}(2790)^{0}$ SIGNAL EXTRACTION}

We extract the number of $\Xi_{c}^{\prime 0}, \Xi_{c}(2645)^{0}$, and $\Xi_{c}(2790)^{0}$ baryons in decays of the type $B^{-} \rightarrow \bar{\Lambda}_{c}^{-} \Xi_{c}^{\prime 0}, B^{-} \rightarrow$ $\bar{\Lambda}_{c}^{-} \Xi_{c}(2645)^{0}$, and $B^{-} \rightarrow \bar{\Lambda}_{c}^{-} \Xi_{c}(2790)^{0}$ by fitting the recoiling mass spectrum $\left(M_{B_{\text {tag }}^{+} \bar{\Lambda}_{c}^{-}}^{\text {rec }}\right)$. We choose $2.5 \mathrm{GeV} / c^{2}<$ $M_{B_{\text {tag }}^{+} \bar{\Lambda}_{c}^{-}}^{\text {rec }}<2.86 \mathrm{GeV} / c^{2}$ as the fit region. To improve the recoil mass resolution, we use $M_{B_{\operatorname{tag}}^{+} \bar{\Lambda}_{c}^{-}}^{\mathrm{rec}} \equiv M_{B_{\operatorname{tag}}^{+\operatorname{si}} \bar{\Lambda}_{c}^{-}}^{\mathrm{mi}}+M_{B_{\mathrm{tag}}^{+}}-$ $m_{B}+M_{\bar{\Lambda}_{c}^{-}}-m_{\bar{\Lambda}_{c}^{-}}$, where $M_{B_{\operatorname{tag}}^{+}}$is the reconstructed and $m_{B}$ is the nominal mass [2] of the $B^{+}$meson and $M_{B_{\text {tag }}^{+} \bar{\Lambda}_{c}^{-}}^{\text {miss }}$ is the invariant mass recoiling against the $\bar{\Lambda}_{c}^{-}$on the signal side, which is calculated using $\sqrt{\left(P_{\text {c.m.s }}-P_{B_{\text {tag }}^{+}}-P_{\bar{\Lambda}_{c}^{-}}\right)^{2}}$ with $P_{\text {c.m.s }}, P_{B_{\text {tag }}^{+}}$, and $P_{\bar{\Lambda}_{c}^{-}}$being 4-momenta of the initial $e^{+} e^{-}$ system, the reconstructed $B_{\text {tag }}^{+}$meson, and the reconstructed $\bar{\Lambda}_{c}^{-}$baryon, respectively.

Figure 1 shows the $\Delta E^{\text {tag }}$ distribution in the $\Xi_{c}^{* 0}$ signal region, i.e., $2.5 \mathrm{GeV} / c^{2}<M_{B_{\mathrm{tag}}^{+} \bar{\Lambda}_{\bar{c}}^{-}}^{\mathrm{rec}}<2.86 \mathrm{GeV} / c^{2}$, after applying all of the above requirements. A double-Gaussian function is used as the signal shape, and the background shape is described by a first-order polynomial. Because of the small sample size, the parameters of the doubleGaussian function are fixed to the values obtained by fitting the signal MC distribution. The fit results are shown as curves in Fig. 1. We take $\left|\Delta E^{\text {tag }}\right|<0.04 \mathrm{GeV}$ as the signal region.

Figure 2 shows the scatter plot of $M_{\bar{\Lambda}_{c}^{-}}$of the signal side in the $\Xi_{c}^{* 0}$ signal region vs $M_{\mathrm{bc}}^{\text {tag }}$ of the $B_{\text {tag }}^{+}$. To check for possible peaking backgrounds, the $M_{\mathrm{bc}}^{\mathrm{tag}}$ and $M_{\bar{\Lambda}_{c}^{-}}$sideband regions are selected as shown in Fig. 2. The normalized contribution from the $M_{\mathrm{bc}}^{\mathrm{tag}}$ and $M_{\bar{\Lambda}_{c}^{-}}$sidebands is estimated using $50 \%$ of the number of events in the blue dashed boxes 


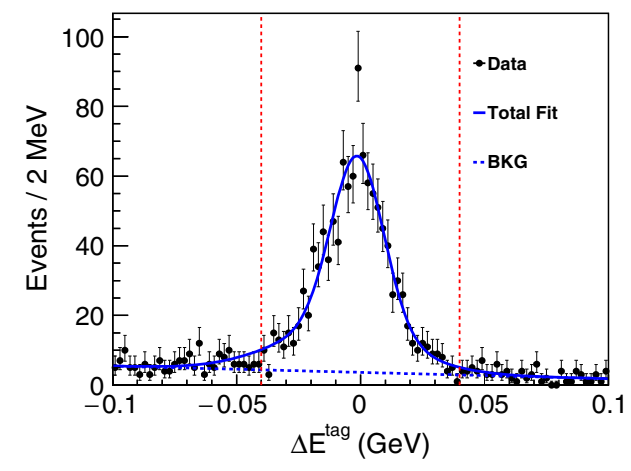

FIG. 1. $\Delta E^{\text {tag }}$ distribution summed over the two reconstructed $\bar{\Lambda}_{c}^{-}$decay modes. The points with error bars represent the data, the solid curve is the best-fit result, and the blue dashed curve is the fitted background. The red dashed lines show the defined $\Delta E^{\text {tag }}$ signal region.

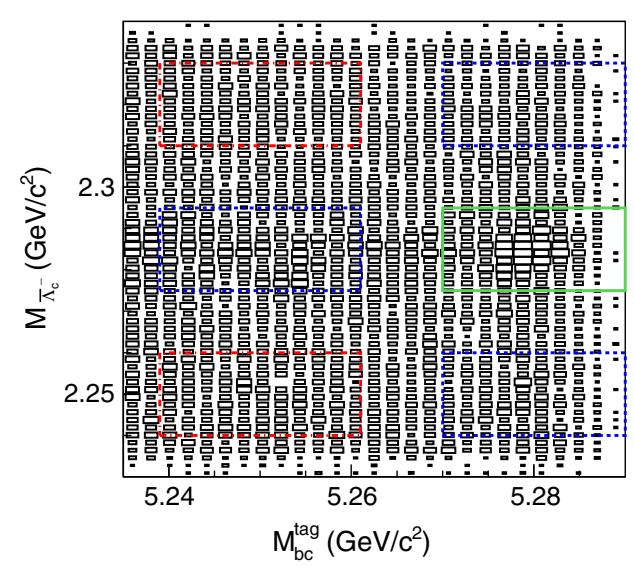

FIG. 2. Scatter plot of $M_{\bar{\Lambda}_{c}^{-}}$of signal side vs $M_{\mathrm{bc}}^{\mathrm{tag}}$ of $B_{\mathrm{tag}}^{+}$in the $\Xi_{c}^{* 0}$ signal region, i.e., $2.5 \mathrm{GeV} / c^{2}<M_{B_{\mathrm{tag}}^{+} \bar{\Lambda}_{c}^{-}}^{\mathrm{rec}}<2.86 \mathrm{GeV} / c^{2}$. The solid box shows the selected signal region, and the blue and red dashed boxes define the $M_{\mathrm{bc}}^{\mathrm{tag}}$ and $M_{\bar{\Lambda}_{c}^{-}}$sidebands described in the text.

minus $25 \%$ of the number of events in the red dashed boxes. Figure 3 shows the $M_{B_{\operatorname{tag}}^{+} \bar{\Lambda}_{c}^{-}}^{\mathrm{rec}}$ distribution in the signal box (points with error bars) and in the sideband boxes (shaded histogram). No peaking background is found in the $M_{\mathrm{bc}}^{\mathrm{tag}}$ and $M_{\bar{\Lambda}_{\bar{c}}^{-}}$sideband events or in the inclusive MC samples of $\Upsilon(4 S) \rightarrow B \bar{B}$ and $e^{+} e^{-} \rightarrow q \bar{q}$ events.

To extract the $\Xi_{c}^{* 0}$ signal yields, an unbinned maximum-likelihood fit to the $M_{B_{\operatorname{tag}}^{+} \bar{\Lambda}_{\bar{c}}^{-}}^{\text {rec }}$ distribution is performed. In this fit, the $\Xi_{c}^{\prime 0}$ signal shape is described by a double-Gaussian function, while the $\Xi_{c}(2645)^{0}$ and $\Xi_{c}(2790)^{0}$ signal shapes are Breit-Wigner (BW) functions convolved with double-Gaussian functions. The background is parametrized by a second-order polynomial function. Due to the limited sample size, the values of the

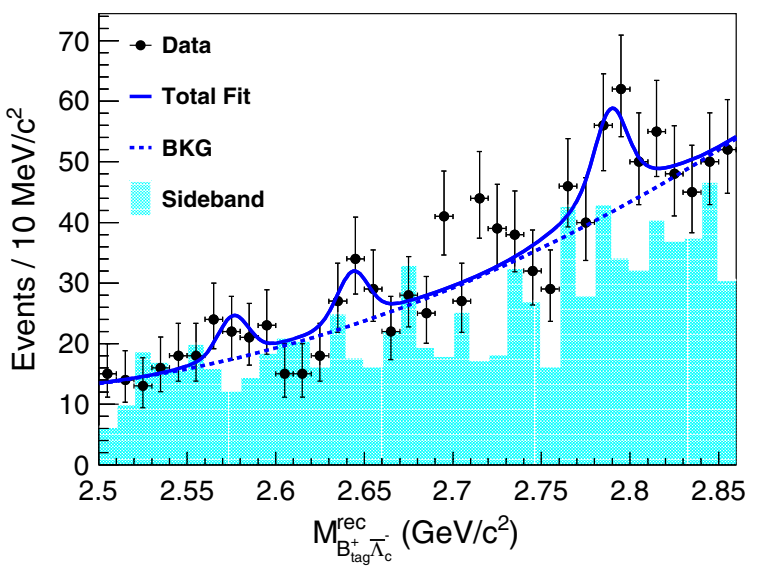

FIG. 3. $M_{B_{\mathrm{tan}}^{+} \bar{\Lambda}_{c}^{-}}^{\mathrm{rec}}$ distribution of selected candidate events. The points with error bars represent the data, the solid blue curve is the best fit, the dashed curve is the fitted total background, and the cyan shaded histogram is the normalized distribution of the $M_{\mathrm{bc}}^{\mathrm{tag}}$ and $M_{\bar{\Lambda}_{c}^{-}}$sideband events (see Fig. 2).

parameters in double-Gaussian functions are fixed to those obtained from the fit to the corresponding signal MC distribution. For $\Xi_{c}(2645)^{0}$ and $\Xi_{c}(2790)^{0}$ signal shapes, the masses and widths of BW functions are fixed to world average values [2]. The fit result is shown in Fig. 3. The difference between the fitted background level and the normalized $M_{\mathrm{bc}}^{\mathrm{tag}}$ and $M_{\bar{\Lambda}_{c}^{-}}$sidebands is due to the contribution from other multibody $B^{-}$decay modes with a $\bar{\Lambda}_{c}^{-}$, for example, $B^{-} \rightarrow K^{-} \Lambda_{c}^{+} \bar{\Lambda}_{c}^{-}$.

The numbers of fitted $\Xi_{c}^{\prime 0}, \Xi_{c}(2645)^{0}$, and $\Xi_{c}(2790)^{0}$ signal events are $N_{\Xi_{c}^{\prime 0}}=17.9 \pm 10.4, N_{\Xi_{c}(2645)^{0}}=24.1 \pm 13.0$, and $N_{\Xi_{c}(2790)^{0}}=59.9 \pm 22.5$ with statistical significances of $1.7 \sigma, 1.9 \sigma$, and $3.1 \sigma$, respectively. Here, the statistical significances are defined as $\sqrt{-2 \ln \left(\mathcal{L}_{0} / \mathcal{L}_{\max }\right)}$, where $\mathcal{L}_{0}$ and $\mathcal{L}_{\max }$ are the maximized likelihoods without and with a signal component, respectively $[20,21]$. The $\Xi_{c}(2790)^{0}$ signal significance becomes $3.0 \sigma$ when systematic uncertainties are included (see below).

Then, the branching fractions are

$$
\begin{aligned}
\mathcal{B}_{\bar{\Lambda}_{c}^{-} \Xi_{c}^{\prime 0}} & =\frac{N_{\Xi_{c}^{\prime 0}}}{2 N_{B^{-}}\left(\varepsilon_{\bar{p} K^{+} \pi^{-}}^{\Xi_{c}^{\prime 0}} \mathcal{B}_{1}+\varepsilon_{\bar{p} K_{s}^{0}}^{\Xi_{c}^{\prime 0}} \mathcal{B}_{2}\right)} \\
& =(3.4 \pm 2.0) \times 10^{-4}, \\
\mathcal{B}_{\bar{\Lambda}_{c}^{-} \Xi_{c}(2645)^{0}} & =\frac{N_{\Xi_{c}(2645)^{0}}}{2 N_{B^{-}}\left(\varepsilon_{\bar{p} K^{+} \pi^{-}}^{\Xi_{c}(2645)^{0}} \mathcal{B}_{1}+\varepsilon_{\bar{p} K_{s}^{0}}^{\Xi_{c}(2645)^{0}} \mathcal{B}_{2}\right)} \\
& =(4.4 \pm 2.4) \times 10^{-4},
\end{aligned}
$$

and 
TABLE I. The detection efficiencies $\varepsilon_{\bar{p} K^{+} \pi^{-}}^{\Xi_{* 0}^{* 0}}$ and $\varepsilon_{\bar{p} K^{0}}^{\Xi_{*}^{* 0}}$ including the $B_{\text {tag }}^{+}$meson for the studied $\bar{\Lambda}_{c}^{-}$decay modes as obtained from MC simulated $B^{-} \rightarrow \bar{\Lambda}_{c}^{-} \Xi_{c}^{* 0}$ processes. All the uncertainties here are statistical only.

\begin{tabular}{lccc}
\hline \hline$\Xi_{c}^{* 0}$ type & $\Xi_{c}^{\prime 0}$ & $\Xi_{c}(2645)^{0}$ & $\Xi_{c}(2790)^{0}$ \\
\hline$\varepsilon_{\bar{p} K^{+} \pi^{-}}^{\Xi_{*}^{* 0}}(\%)$ & $0.09 \pm 0.003$ & $0.09 \pm 0.003$ & $0.09 \pm 0.003$ \\
$\varepsilon_{\bar{p} K_{s}^{* 0}}^{\Xi_{0}^{* 0}}(\%)$ & $0.13 \pm 0.003$ & $0.14 \pm 0.004$ & $0.15 \pm 0.004$ \\
\hline \hline
\end{tabular}

$$
\begin{aligned}
\mathcal{B}_{\bar{\Lambda}_{c}^{-} \Xi_{c}(2790)^{0}} & =\frac{N_{\Xi_{c}(2790)^{0}}}{2 N_{B^{-}}\left(\varepsilon_{\bar{p} K^{+} \pi^{-}}^{\Xi_{c}(2790)^{0}} \mathcal{B}_{1}+\varepsilon_{\bar{p} K_{s}^{0}}^{\Xi_{c}(2790)^{0}} \mathcal{B}_{2}\right)} \\
& =(1.1 \pm 0.4) \times 10^{-3},
\end{aligned}
$$

where $\mathcal{B}_{\bar{\Lambda}_{c}^{-} \Xi_{c}^{\prime 0}}=\mathcal{B}\left(B^{-} \rightarrow \bar{\Lambda}_{c}^{-} \Xi_{c}^{\prime 0}\right), \mathcal{B}_{\bar{\Lambda}_{c}^{-} \Xi_{c}(2645)^{0}}=\mathcal{B}\left(B^{-} \rightarrow\right.$ $\left.\bar{\Lambda}_{c}^{-} \Xi_{c}(2645)^{0}\right), \quad \mathcal{B}_{\bar{\Lambda}_{c}^{-} \Xi_{c}(2790)^{0}}=\mathcal{B}\left(B^{-} \rightarrow \bar{\Lambda}_{c}^{-} \Xi_{c}(2790)^{0}\right)$, $N_{B^{-}}=N_{\Upsilon(4 S)} \mathcal{B}\left(\Upsilon(4 S) \rightarrow B^{+} B^{-}\right)$with $N_{\Upsilon(4 S)}$ being the number of accumulated $\Upsilon(4 S)$ events. We use a value of 0.514 for $\mathcal{B}\left(\Upsilon(4 S) \rightarrow B^{+} B^{-}\right)[2] ; \mathcal{B}_{1}=\mathcal{B}\left(\bar{\Lambda}_{c}^{-} \rightarrow \bar{p} K^{+} \pi^{-}\right)$, $\mathcal{B}_{2}=\mathcal{B}\left(\bar{\Lambda}_{c}^{-} \rightarrow \bar{p} K_{S}^{0}\right) \mathcal{B}\left(K_{S}^{0} \rightarrow \pi^{+} \pi^{-}\right), \quad \mathcal{B}\left(\bar{\Lambda}_{c}^{-} \rightarrow \bar{p} K^{+} \pi^{-}\right)$, $\mathcal{B}\left(\bar{\Lambda}_{c}^{-} \rightarrow \bar{p} K_{S}^{0}\right)$, and $\mathcal{B}\left(K_{S}^{0} \rightarrow \pi^{+} \pi^{-}\right)$are the branching fractions of $\bar{\Lambda}_{c}^{-} \rightarrow \bar{p} K^{+} \pi^{-}, \bar{\Lambda}_{c}^{-} \rightarrow \bar{p} K_{S}^{0}$, and $K_{S}^{0} \rightarrow \pi^{+} \pi^{-}$ [2], respectively. $\varepsilon_{\bar{p} K^{+} \pi^{-}}^{\Xi_{* 0}^{* 0}}$ and $\varepsilon_{\bar{p} K_{s}^{0}}^{\Xi_{s}^{* 0}}$ are the detection efficiencies of different $\bar{\Lambda}_{c}^{-}$decay modes which are obtained from fits to the signal MC samples and are listed in Table I.

Since the statistical significances of $\Xi_{c}^{\prime 0}$ and $\Xi_{c}(2645)^{0}$ are less than $3 \sigma$, Bayesian upper limits at $90 \%$ credibility level (C.L.) [22] assuming a uniform prior probability for $\mathcal{B}\left(B^{-} \rightarrow \bar{\Lambda}_{c}^{-} \Xi_{c}^{\prime 0}\right)$ and $\mathcal{B}\left(B^{-} \rightarrow \bar{\Lambda}_{c}^{-} \Xi_{c}(2645)^{0}\right)\left(\right.$ denoted $\left.\mathcal{B}^{\text {up }}\right)$ are determined by solving the equation

$$
\int_{0}^{\mathcal{B}^{\text {up }}} \mathcal{L}(\mathcal{B}) d \mathcal{B} / \int_{0}^{1} \mathcal{L}(\mathcal{B}) d \mathcal{B}=0.9
$$

where

$$
\mathcal{B}=N_{\Xi_{c}^{* 0}} / 2 N_{B^{-}}\left(\varepsilon_{\bar{p} K^{+} \pi^{-}}^{\Xi_{c}^{* 0}} \mathcal{B}_{1}+\varepsilon_{\bar{p} K_{s}^{0}}^{\Xi_{c}^{* 0}} \mathcal{B}_{2}\right)
$$

is the assumed branching fraction for $B^{-} \rightarrow \bar{\Lambda}_{c}^{-} \Xi_{c}^{\prime 0}$ or $B^{-} \rightarrow$ $\bar{\Lambda}_{c}^{-} \Xi_{c}(2645)^{0}$ and $\mathcal{L}(\mathcal{B})$ is the corresponding likelihood of the data. $N_{\Xi_{c}^{* 0}}$ is the fitted signal yield of $\Xi_{c}^{\prime 0}$ or $\Xi_{c}(2645)^{0}$. Taking into account the systematic uncertainty discussed below, the likelihoods are convolved with a Gaussian function of which the width equals the corresponding total systematic uncertainty. The $90 \%$ C.L. upper limits with systematic uncertainties included on $\mathcal{B}\left(B^{-} \rightarrow \bar{\Lambda}_{c}^{-} \Xi_{c}^{\prime 0}\right)$ and $\mathcal{B}\left(B^{-} \rightarrow \bar{\Lambda}_{c}^{-} \Xi_{c}(2645)^{0}\right)$ are determined to be $6.5 \times 10^{-4}$ and $7.9 \times 10^{-4}$, respectively.

Table II summarizes the fitted results, branching fractions, and statistical significances for $B^{-} \rightarrow \bar{\Lambda}_{c}^{-} \Xi_{c}^{\prime 0}, B^{-} \rightarrow$ $\bar{\Lambda}_{c}^{-} \Xi_{c}(2645)^{0}$, and $B^{-} \rightarrow \bar{\Lambda}_{c}^{-} \Xi_{c}(2790)^{0}$. The uncertainties shown are statistical only.

\section{SYSTEMATIC UNCERTAINTIES}

There are several sources of systematic uncertainties for the branching-fraction measurements as listed in Table III, including the reconstruction efficiency-related sources, the fit uncertainty, the $\Lambda_{c}$ decay branching fractions, the $B$ meson tag efficiency, and the total number of $B \bar{B}$ events.

The reconstruction efficiency-related uncertainties include those for tracking efficiency $(0.35 \%$ per track), particle identification efficiency ( $1.44 \%$ per kaon, $0.86 \%$ per pion, and range from $2.13 \%$ to $3.13 \%$ per proton), as well as momentum-weighted $K_{S}^{0}$ selection efficiency $(1.1 \%)$ [23]. Here, the systematic uncertainty due to the $K_{S}^{0}$ selection depends on the $K_{S}^{0}$ momentum and was determined using a control sample of $D^{*+} \rightarrow D^{0}\left(K_{S}^{0} \pi^{0}\right) \pi^{+}$. For the three branching-fraction measurements, the individual reconstruction efficiency-related uncertainties from two

TABLE II. Summary of the fitted signal yields $\left(N_{\text {sig }}\right)$, branching fractions (90\% C.L. upper limits), and statistical signal significances $(\sigma)$ for $B^{-} \rightarrow \bar{\Lambda}_{c}^{-} \Xi_{c}^{\prime 0}, B^{-} \rightarrow \bar{\Lambda}_{c}^{-} \Xi_{c}(2645)^{0}$, and $B^{-} \rightarrow \bar{\Lambda}_{c}^{-} \Xi_{c}(2790)^{0}$. All the uncertainties here are statistical only.

\begin{tabular}{lccc}
\hline \hline & $N_{\text {sig }}$ & $\mathcal{B}\left(B^{-} \rightarrow \bar{\Lambda}_{c}^{-} \Xi_{c}^{* 0}\right)[$ Upper limit] & Significance $(\sigma)$ \\
\hline$\Xi_{c}^{\prime 0}$ & $17.9 \pm 10.4$ & $(3.4 \pm 2.0) \times 10^{-4}\left[6.5 \times 10^{-4}\right]$ & 1.7 \\
$\Xi_{c}(2645)^{0}$ & $24.1 \pm 13.0$ & $(4.4 \pm 2.4) \times 10^{-4}\left[7.9 \times 10^{-4}\right]$ & 1.9 \\
$\Xi_{c}(2790)^{0}$ & $59.9 \pm 22.5$ & $(1.1 \pm 0.4) \times 10^{-3}$ & 3.1 \\
\hline \hline
\end{tabular}

TABLE III. Summary of the relative systematic uncertainties on the branching-fraction measurements $(\%)$ for $B^{-} \rightarrow \bar{\Lambda}_{c}^{-} \Xi_{c}^{\prime 0}$, $B^{-} \rightarrow \bar{\Lambda}_{c}^{-} \Xi_{c}(2645)^{0}$, and $B^{-} \rightarrow \bar{\Lambda}_{c}^{-} \Xi_{c}(2790)^{0}$.

\begin{tabular}{lccccccc}
\hline \hline Observable & Efficiency & Fit & $\bar{\Lambda}_{c}^{-}$decays & $B_{\text {tag }}$ & $N_{B^{ \pm}}$ & Total & Measured value \\
\hline $\mathcal{B}\left(B^{-} \rightarrow \bar{\Lambda}_{c}^{-} \Xi_{c}^{\prime 0}\right)$ & 3.1 & 10.0 & 5.5 & 4.2 & 1.8 & 12.6 & $(3.4 \pm 2.0 \pm 0.4) \times 10^{-4}$ \\
$\mathcal{B}\left(B^{-} \rightarrow \bar{\Lambda}_{c}^{-} \Xi_{c}(2645)^{0}\right)$ & 3.3 & 8.1 & 5.5 & 4.2 & 1.8 & 11.3 & $(4.4 \pm 2.4 \pm 0.5) \times 10^{-4}$ \\
$\mathcal{B}\left(B^{-} \rightarrow \bar{\Lambda}_{c}^{-} \Xi_{c}(2790)^{0}\right)$ & 3.5 & 11.0 & 5.5 & 4.2 & 1.8 & 13.6 & $(1.1 \pm 0.4 \pm 0.2) \times 10^{-3}$ \\
\hline \hline
\end{tabular}


different $\bar{\Lambda}_{c}^{-}$decay channels are added, linearly weighted by the product of the detection efficiency and $\bar{\Lambda}_{c}^{-}$partial decay width. Then, those uncertainties are summed in quadrature to be the final uncertainties related to the efficiency of the reconstruction, yielding $3.1 \%$ to $3.5 \%$, depending on the specific decay mode.

We estimate the systematic uncertainties associated with the fit by changing the order of the background polynomial, by changing the range of the fit, and by enlarging the mass resolution by $10 \%$. The observed deviations are taken as systematic uncertainties. The masses of $\Xi_{c}(2790)^{0}$ and $\Xi_{c}(2815)^{0}$ are rather close, and no $\Xi_{c}(2815)^{0}$ signal peak can be seen. The $\Xi_{c}(2815)^{0}$ signal significance is only $0.4 \sigma$ if it is added in the fit. So, we take the difference of the number of $\Xi_{c}(2790)^{0}$ signal events as the systematic uncertainty due to the possible contribution of $\Xi_{c}(2815)^{0}$ from $B^{-} \rightarrow \bar{\Lambda}_{c}^{-} \Xi_{c}(2815)^{0}$. Finally, all the above uncertainties are summed in quadrature, and the sums are taken as the systematic uncertainties associated with the fit.

Uncertainties for the $\bar{\Lambda}_{c}^{-}$decay branching fractions are due to $\mathcal{B}\left(\bar{\Lambda}_{c}^{-} \rightarrow f_{i}\right)=\Gamma_{i} \times \mathcal{B}\left(\bar{\Lambda}_{c}^{-} \rightarrow \bar{p} K^{+} \pi^{-}\right)$; here, $\Gamma_{i}=\mathcal{B}\left(\bar{\Lambda}_{c}^{-} \rightarrow f_{i}\right) / \mathcal{B}\left(\bar{\Lambda}_{c}^{-} \rightarrow \bar{p} K^{+} \pi^{-}\right)$, and $f_{i}$ denotes the different $\bar{\Lambda}_{c}^{-}$decay modes. Uncertainties on $\mathcal{B}\left(\bar{\Lambda}_{c}^{-} \rightarrow\right.$ $\left.\bar{p} K^{+} \pi^{-}\right)$and $\Gamma\left(\bar{\Lambda}_{c}^{-} \rightarrow \bar{p} K_{s}^{0}\right) / \Gamma\left(\bar{\Lambda}_{c}^{-} \rightarrow \bar{p} K^{+} \pi^{-}\right)$are taken from Ref. [2]. The final uncertainties on the two $\bar{\Lambda}_{c}^{-}$partial decay widths are summed in quadrature with the detection efficiency as a weighting factor. The uncertainty due to the $B$-meson tagging efficiency is $4.2 \%$ [24]. The uncertainty on $\mathcal{B}\left(\Upsilon(4 S) \rightarrow B^{+} B^{-}\right)$is $1.2 \%$ [2]. The systematic uncertainty on $N_{\Upsilon(4 S)}$ is $1.37 \%$. The sources of uncertainty summarized in Table III are assumed to be independent and thus are added in quadrature to obtain the total systematic uncertainty.

\section{CONCLUSION}

Using the $711 \mathrm{fb}^{-1}$ data sample taken at the $\Upsilon(4 S)$ resonance that corresponds to $(772 \pm 11) \times 10^{6} B \bar{B}$ pairs accumulated with the Belle detector at the KEKB asymmetric-energy $e^{+} e^{-}$collider, we present the first measurements of the branching fractions of the decays $B^{-} \rightarrow \bar{\Lambda}_{c}^{-} \Xi_{c}^{\prime \prime}$, $B^{-} \rightarrow \bar{\Lambda}_{c}^{-} \Xi_{c}(2645)^{0}$, and $B^{-} \rightarrow \bar{\Lambda}_{c}^{-} \Xi_{c}(2790)^{0}$ with $\Xi_{c}^{* 0} \rightarrow$ anything and the $\bar{\Lambda}_{c}^{-}$candidates reconstructed via their $\bar{p} K^{+} \pi^{-}$and $\bar{p} K_{S}^{0}$ decay modes. The branching fractions are measured to be

$$
\begin{gathered}
\mathcal{B}\left(B^{-} \rightarrow \bar{\Lambda}_{c}^{-} \Xi_{c}^{\prime 0}\right)=(3.4 \pm 2.0 \pm 0.4) \times 10^{-4}, \\
\mathcal{B}\left(B^{-} \rightarrow \bar{\Lambda}_{c}^{-} \Xi_{c}(2645)^{0}\right)=(4.4 \pm 2.4 \pm 0.5) \times 10^{-4},
\end{gathered}
$$

and

$$
\mathcal{B}\left(B^{-} \rightarrow \bar{\Lambda}_{c}^{-} \Xi_{c}(2790)^{0}\right)=(1.1 \pm 0.4 \pm 0.2) \times 10^{-3},
$$

with statistical significances of $1.7 \sigma, 1.9 \sigma$, and $3.1 \sigma$, respectively. Since the statistical significances are less than
$3 \sigma$ for $B^{-} \rightarrow \bar{\Lambda}_{c}^{-} \Xi_{c}^{\prime 0}$ and $B^{-} \rightarrow \bar{\Lambda}_{c}^{-} \Xi_{c}(2645)^{0}$, the $90 \%$ C.L. upper limits on $\mathcal{B}\left(B^{-} \rightarrow \bar{\Lambda}_{c}^{-} \Xi_{c}^{\prime 0}\right)$ and $\mathcal{B}\left(B^{-} \rightarrow \bar{\Lambda}_{c}^{-} \Xi_{c}(2645)^{0}\right)$ are determined to be $6.5 \times 10^{-4}$ and $7.9 \times 10^{-4}$, respectively, with systematic uncertainties included.

\section{ACKNOWLEDGMENTS}

We thank the KEKB group for the excellent operation of the accelerator; the KEK cryogenics group for the efficient operation of the solenoid; and the KEK computer group, and the Pacific Northwest National Laboratory Environmental Molecular Sciences Laboratory computing group for strong computing support; and the National Institute of Informatics and Science Information NETwork 5 for valuable network support. We acknowledge support from the Ministry of Education, Culture, Sports, Science, and Technology (MEXT) of Japan, the Japan Society for the Promotion of Science (JSPS), and the Tau-Lepton Physics Research Center of Nagoya University; the Australian Research Council including Grants No. DP180102629, No. DP170102389, No. DP170102204, No. DP150103061, and No. FT130100303; Austrian Science Fund (FWF); the National Natural Science Foundation of China under Contracts No. 11435013, No. 11475187, No. 11521505, No. 11575017, No. 11675166, No. 11705209, No. 11761141009, and No. 11975076; Key Research Program of Frontier Sciences, Chinese Academy of Sciences (CAS), Grant No. QYZDJ-SSW-SLH011; the CAS Center for Excellence in Particle Physics (CCEPP); the Shanghai Pujiang Program under Grant No. 18PJ1401000; the Ministry of Education, Youth and Sports of the Czech Republic under Contract No. LTT17020; the Carl Zeiss Foundation, the Deutsche Forschungsgemeinschaft, the Excellence Cluster Universe, and the VolkswagenStiftung; the Department of Science and Technology of India; the Istituto Nazionale di Fisica Nucleare of Italy; National Research Foundation (NRF) of Korea Grants No. 2016R1D1A1B01010135, No. 2016R1D1A1B02012900, No. 2018R1A2B3003643, No. 2018R1A6A1A06024970, No. 2018R1D1A1B07047294, No. 2019K1A3A7A09033840, and No. 2019R1I1A3A01058933; Radiation Science Research Institute, Foreign Large-size Research Facility Application Supporting project, the Global Science Experimental Data Hub Center of the Korea Institute of Science and Technology Information, and KREONET/GLORIAD; the Polish Ministry of Science and Higher Education and the National Science Center; the Ministry of Science and Higher Education of the Russian Federation, Grant No. 14.W03.31.0026; the Slovenian Research Agency; Ikerbasque, Basque Foundation for Science, Spain; the Swiss National Science Foundation; the Ministry of Education and the Ministry of Science and Technology of Taiwan; and the United States Department of Energy and the National Science Foundation. 
[1] N. Isgur and M. B. Wise, Phys. Rev. Lett. 66, 1130 (1991).

[2] M. Tanabashi et al. (Particle Data Group), Phys. Rev. D 98, 030001 (2018).

[3] D. Ebert, R. N. Faustov, and V. O. Galkin, Phys. Rev. D 72 , 034026 (2005).

[4] C. Semay, F. Buisseret, and F. Stancu, Phys. Rev. D 78, 076003 (2008).

[5] O. Romanets, L. Tolos, C. Garcia-Recio, J. Nieves, L. L. Salcedo, and R. G. E. Timmermans, Phys. Rev. D 85, 114032 (2012).

[6] J. R. Zhang and M. Q. Huang, Phys. Rev. D 78, 094015 (2008).

[7] H. X. Chen, W. Chen, Q. Mao, A. Hosaka, X. Liu, and S. L. Zhu, Phys. Rev. D 91, 054034 (2015).

[8] J. Yelton et al. (Belle Collaboration), Phys. Rev. D 94, 052011 (2016).

[9] Y. B. Li et al. (Belle Collaboration), Phys. Rev. Lett. 122, 082001 (2019).

[10] M. Feindt, F. Keller, M. Kreps, T. Kuhr, S. Neubauer, D. Zander, and A. Zupanc, Nucl. Instrum. Methods Phys. Res., Sect. A 654, 432 (2011).

[11] Inclusion of charge-conjugate states is implicit unless otherwise stated.

[12] A. Abashian et al. (Belle Collaboration), Nucl. Instrum. Methods Phys. Res., Sect. A 479, 117 (2002); also, see the detector section in J. Brodzicka et al., Prog. Theor. Exp. Phys. 2012, 04D001 (2012).

[13] S. Kurokawa and E. Kikutani, Nucl. Instrum. Methods Phys. Res., Sect. A 499, 1 (2003), and other papers included in this volume; T. Abe et al., Prog. Theor. Exp. Phys. 2013, 03A001 (2013), and references therein.

[14] D. J. Lange, Nucl. Instrum. Methods Phys. Res., Sect. A 462, 152 (2001).

[15] T. Sjöstrand, P. Edén, C. Friberg, L. Lönnblad, G. Miu, S. Mrenna, and E. Norrbin, Comput. Phys. Commun. 135, 238 (2001).

[16] R. Brun et al., GEANT 3: User's guide Geant 3.10, Geant 3.11, CERN Report No. DD/EE/84-1, 1984.

[17] E. Nakano, Nucl. Instrum. Methods Phys. Res., Sect. A 494, 402 (2002).

[18] M. Feindt and U. Kerzel, Nucl. Instrum. Methods Phys. Res., Sect. A 559, 190 (2006).

[19] H. Nakano, Ph.D Thesis, Tohoku University, 2014, Chapter 4, unpublished, https://tohoku.repo.nii.ac.jp/?action=pages_ view_main\&active_action=repository_view_main_item_ detail\&item_id=70563\&item_no=1\&page_id=33\&block_ id=38 (English).

[20] S. S. Wilks, Ann. Math. Stat. 9, 60 (1938).

[21] G. Cowan, K. Cranmer, E. Gross, and O. Vitells, Eur. Phys. J. C 73, 2501 (2013).

[22] In common high energy physics usage, this Bayesian interval has been reported as a "confidence interval," which is a frequentist-statistics term.

[23] N. Dash et al. (Belle Collaboration), Phys. Rev. Lett. 119, 171801 (2017).

[24] A. Sibidanov et al. (Belle Collaboration), Phys. Rev. D 88, 032005 (2013). 